\title{
Nebenwirkungen von Psychopharmaka: Tipps für die Praxis
}

Anna Grunze, Rajnish Mago, Heinz Grunze

\author{
Psychopharmaka sind hochwirksame, aber auch mit Nebenwirkungen behaftete Medika- \\ mente, die für viele Patienten unverzichtbar sind. Der Umgang mit Nebenwirkungen - \\ Zuwarten, Dosisreduktion, Medikamentenwechsel, Zugabe eines „Antidots“ sowie spe- \\ zielle Verhaltensmaßnahmen - hängt von ihrer Art, Schwere und dem Wunsch des Pa- \\ tienten ab. Dieser Beitrag soll Arzt und Patient Entscheidungshilfen bei häufigen und be- \\ einträchtigenden Nebenwirkungen bieten.
}

\section{Psychopharmakotherapie}

Unerwünschte Arzneimittelwirkungen (UAW) von Psychopharmaka - in erster Linie Antidepressiva und Antipsychotika (Neuroleptika) - stellen nicht nur die Adhärenz des Patienten auf eine Belastungsprobe. Wenn nicht adäquat darauf eingegangen wird, können sie auch das therapeutische Verhältnis beschädigen [1]. Die Art, wie UAW angegangen werden, hängt nicht zuletzt von der Einstellung des Behandlers gegenüber psychischen Erkrankungen ab: Werden sie als Befindlichkeitsstörungen ohne hohen Krankheitswert gesehen, werden Psychopharmaka gerne als „Life Style Drugs“ betrachtet und abgesetzt. Ein unreflektiertes Absetzen der Medikation kommt auch vor, wenn Psychopharmaka vom Arzt aus mangelnder Erfahrung - oder vom Patienten als etwas Fragwürdiges oder potenziell Gefährliches wahrgenommen werden. Nicht unerheblich ist dabei der Einfluss neuer Medien, in denen nicht nur Informationen, sondern auch Ideologien an den Patienten herangetragen werden - ein Problem, das auch in anderen medizinischen Bereichen, z. B. bei Impfungen, auftritt.

\section{HINTERGRUND}

Es sollte stets bedacht werden: Psychiatrische Erkrankungen führen in einem weitaus höheren Maße als viele somatische Erkrankungen - bei denen die medikamentöse Therapie traditionell nicht infrage gestellt wird - zu andauernder Behinderung und deutlich verkürzter Lebenserwartung.

Andauernde Behinderung [2] und deutlich verkürzte Lebenserwartung sind Charakteristika schwerer psychischer Erkrankungen. Ursächlich sind dabei nicht allein der Suizid, sondern ebenso Herz-Kreislauf-Erkrankungen, Neoplasien und Unfälle [3]. Umgekehrt ist gut belegt, dass z. B. das Mortalitätsrisiko bei kardialen Erkrankungen bei gleichzeitigem Vorliegen einer Depression signifikant ansteigt [4] und die Behandlung erschwert wird [5, 6].

\section{Merke}

Therapieverzicht stellt bei schweren psychiatrischen Erkrankungen im Regelfall keine Option dar.

Bezüglich der oft geäußerten Skepsis zur Wirksamkeit von Psychopharmaka ist zu betonen: Es handelt sich hier um eine der wirkungsvollsten Medikamentengruppen mit einer Number needed to treat (NNT) im einstelligen Bereich [7]. Zum Vergleich: Die NNT für Acetylsalicylsäure zur Sekundärprophylaxe nach Herzinfarkt beträgt 67 . Hochwirksame Medikamente bedeuten aber oft auch ein höheres Maß an UAW.

\section{Ursachen von UAW}

Manche Patienten sind deutlich empfänglicher für UAW. Die Ursachen können psychischer Natur sein, z. B. Panikstörungen oder generalisierte Angsterkrankung. Aber auch eine veränderte Pharmakokinetik kann ursächlich sein, z. B. durch Interaktionen mit anderen Medikamenten oder verlangsamten Abbau („Poor Metabolizer“ aufgrund eines entsprechenden Cytochrom-P450-Polymorphismus, zumeist CYP 2D6 Poor Metabolizer).

\section{Merke}

Im Falle eines „Poor-Metabolizer“-Status sind sehr langsame Aufdosierung, reduzierte Dosis und - wenn verfügbar - Serumspiegelkontrollen (Therapeutic Drug Monitoring, TDM) angezeigt.

Leicht können aber auch im Rahmen eines abrupten Therapiewechsels, insbesondere von einem Antidepressivum zu einem Anderen, vegetative Symptome, als Nebenwirkung des neuen Medikamentes, fehlinterpretiert werden. Sie müssen von Absetzsymptomen („Discontinuation Symptoms“) des vorherigen Antidepressivums unterschieden werden $[8,9]$. 
Einige UAW von Psychopharmaka treten meist nur zeitlich begrenzt zu Therapiebeginn auf, z. B. Übelkeit bei Serotonin-Wiederaufnahmehemmern (SSRI). Dann kann ein Zuwarten, sofern für den Patienten akzeptabel, gerechtfertigt sein.

\section{Merke}

Bei Persistenz von UAW sind deren Häufigkeit, Schwere und Auswirkungen auf das tägliche Leben (z. B. eingeschränkte Fahrtauglichkeit) detailliert abzuklären.

\section{Maßnahmen bei UAW}

Meist wird eine Dosisadaption, ein Wechsel der Galenik (z. B. auf Retardpräparate) oder eine Veränderung der Einnahmemodalität (z. B. Einmaldosis zur Nacht bei sedierender Nebenwirkung) erforderlich sein. Ergibt sich hierdurch keine Besserung, so bleiben im Wesentlichen 3 Alternativen:

- ein Wechsel auf ein Alternativpräparat,

- der Versuch einer Abmilderung der UAW durch eine Zusatzmedikation als „Antidot“ oder

- verhaltensmodifizierende Maßnahmen.

Zielgruppe sind insbesondere Patienten, die von einer Therapie sichtbar profitieren und bei denen ein Therapiewechsel riskant erscheint oder bei denen es keine oder nur schlechtere Alternativen gibt.

Die nachfolgenden Ausführungen beziehen sich ausdrücklich auf häufige UAW, die die Lebensqualität beeinträchtigen. Sie gelten aber nicht für schwere UAW, die tödlich oder potenziell lebensgefährlich sind oder zu Organschäden mit der Konsequenz bleibender Behinderung führen können (Definition „Severe adverse drug reaction“ der EMA, CPMP/ICH/377/95, Juni 1995). Hinsichtlich des Auftretens dieser selteneren und schweren Arzneimittelnebenwirkungen sei auf Standardlehrbücher der Psychopharmakologie verwiesen (z. B. [10 - 12]).

\section{Häufige UAW unter Psychopharmaka}

- Tab. 1 fasst die häufigsten nicht schweren UAW gängiger Psychopharmaka zusammen. Einige davon sind dosisabhängig - insbesondere Kleinhirnzeichen unter Lithium oder Valproat oder andere ZNS-Nebenwirkungen wie z. B. Doppelbilder unter Carbamazepin. Sie sind somit Hinweis auf eine beginnende Intoxikation mit der Konsequenz, die Dosis zu reduzieren bzw. das Medikament auszusetzen - allerdings können diese UAW auch unter Spiegeln im Normbereich auftreten. Andere UAW, wie z. B. Übelkeit und Kopfschmerz, sind nur bedingt dosisabhängig. Hier sind auch andere gegen die UAW gerichtete Therapiestrategien denkbar.

\section{Cave}

Beim Einsatz von Medikamenten zur Abmilderung von Nebenwirkungen handelt es sich meist um einen „Offlabel“-Gebrauch. Daher müssen Nutzen und Risiken noch stärker abgewogen und dokumentiert werden und der Patient ist ausführlich zu informieren.

Im Folgenden möchten wir teils aus der Literatur, teils aus der eigenen klinischen Erfahrung gewonnene Strategien zur Beherrschung von häufigen UAW vorstellen - insbesondere für die Situation, wenn eine Dosisreduktion oder ein Absetzen des Medikamentes nicht infrage kommt.

\section{Übelkeit (Nausea)}

Übelkeit ist eine der häufigsten UAW zu Therapiebeginn, insbesondere bei

- serotonerg wirksamen Medikamenten, wie SerotoninWiederaufnahmehemmern (SSRI, via Stimulation von 5-HT3-Rezeptoren) oder

- dopaminergen Medikamenten, z. B. Pramipexol oder L-Dopa, aber auch z. B. Bupropion (via Stimulation von D2-Rezeptoren der Area postrema im Hirnstamm).

Langsame Titration und Einnahme nach den Mahlzeiten kann das Risiko medikamenteninduzierter Übelkeit halbieren [13]. Patienten mit vorbestehenden gastrointestinalen Problemen, z. B. einer Refluxoösophagitis, scheinen für Übelkeit anfälliger zu sein. Die gleichzeitige Gabe eines Protonenpumpeninhibitors, z. B. Omeprazol, kann hilfreich sein.

Cave

Omeprazol erhöht durch Hemmung des CYP 2C19 auch die Serumspiegel von Citalopram und Escitalopram entsprechend sollten diese Medikamente geringer dosiert werden.

Auch ein Löffel Erdnussbutter vor der Medikation kann helfen, eine Magenschleimhautirritation zu vermindern, falls das Medikament auf nüchternen Magen genommen werden soll. Dies gilt z. B. für Quetiapin in retardierter Form. Quetiapin soll nicht mit einer vollen Mahlzeit eingenommen werden, da dadurch die Freisetzung beschleunigt wird. Dies hat bis zu 50 \% höhere Plasmaspitzenkonzentrationen und damit verbunden deutlichere Nebenwirkungen zur Folge. Auch in Deutschland ist Erdnussbutter mittlerweile selbst bei Discountern erhältlich. Ein Teelöffel Erdnussbutter hat ca. 30 kcal, also weniger als z. B. Butter in vergleichbarer Menge. Erdnussbutter ist schmackhaft und enthält zudem ein hohes Maß an ungesättigten Fettsäuren, Magnesium und Vitamin E. 
- Tab. 1 Häufigere (>1\% der Patienten), nicht schwerwiegende Nebenwirkungen von gängigen Psychopharmaka.

\section{Medikamente}

\section{Antidepressiva}

SSRI (Citalopram, Escitalopram, Sertralin, Fluoxetin, Paroxetin, Fluvoxamin)

SNRI (Venlafaxin, Duloxetin)

DNRI (Bupropion)

TZA (z. B. Amitriptylin, Clomipramin, Imipramin, Doxepin, Maprotilin, Mianserin)

NaSSA (Mirtazapin)

MMBA (Vortioxetin)

MAO-I (Moclobemid, Tranylcypromin)

MSAD (Agomelatin)

pflanzliche Antidepressiva: Johanniskrautextrakte $>300 \mathrm{mg}$ Tagesdosis

\section{Antipsychotika (Neuroleptika)}

THA (z. B. Perphenazin, Fluphenazin, Haloperidol, Benperidol)

TMNA (z. B. Chlorpromazin, Perazin, Zuclopenthixol, Promethazin, Levomepromazin, Thioridazin, Promazin)

AAD (Clozapin, Zotepin, Olanzapin, Quetiapin, Asenapin)

AAB (Risperidon, Ziprasidon, Paliperidon, Aripiprazol)

AABA (Sulpirid, Amisulprid)
Nebenwirkungen zu Therapiebeginn

Übelkeit, Erbrechen, Diarrhö, vermehrtes Schwitzen, Kopfschmerzen, Appetit- und Gewichtsveränderungen, Unruhe, Schlafstörungen, sexuelle Funktionsstörungen wie SSRI, zusätzlich arterielle Hypertonie bei höheren Dosierungen

Schlafstörungen, Tremor, Kopfschmerzen, Schwindel, Unruhe, trockener Mund, Übelkeit und Erbrechen, Appetitlosigkeit, Bauchschmerzen, Obstipation, Schwitzen, arterielle Hypertonie, selten Priapismus

Sedierung, Schwindel, Tremor, verschwommenes Sehen, orthostatische Dysregulation, Tachykardie, Hitzewallungen, Obstipation, Harnverhalt, Mundtrockenheit, verstopfte Nase, Veränderung des Augeninnendrucks

Sedierung, Kopfschmerzen, Obstipation,

Mundtrockenheit, Übelkeit, Diarrhö, periphere Ödeme, orthostatische Hypotonie

wie SSRI, geringere Inzidenz sexueller Dysfunktionen

Appetitlosigkeit, Schlafstörungen, Unruhe, Schwindel, Kopfschmerzen, Parästhesien, Geschmacksstörungen, Hypotonie, Hitzegefühl, Mundtrockenheit, Übelkeit, Diarrhö und Obstipation

Kopfschmerzen, Übelkeit, Schwindel, Unruhe

Müdigkeit, Unruhe, seltener gastrointestinale Symptome, Fotosensibilisierung, Parästhesien

Parkinsonoid: Tremor, Rigor, Hypokinese; Akathisie, Dyskinesien, okulogyrische Krise; Sehstörungen, erektile Dysfunktion, arterielle Hypertonie, Galaktorrhö, Gewichtsverlust, Schwindel, Kopfschmerzen, Somnolenz

Schlafstörungen, verstopfte Nase, Veränderung des Augeninnendrucks, Schwitzen, vermehrtes Durstgefühl, Mundtrockenheit, Galaktorrhö, Parkinsonoid, Hypotonie, orthostatische Regulationsstörungen

Schläfrigkeit, orthostatische Hypotonie, Obstipation, Harnverhalt, Mundtrockenheit oder Speichelfluss (Clozapin), gelegentlich Symptome eines erhöhten Prolaktinspiegels: Amenorrhö, Gynäkomastie, Galaktorrhö, sexuelle Dysfunktion

Parkinsonoid, Akathisie; Risperdon, Paliperidon und Ziprasidon: häufig Symptome eines erhöhten Prolaktinspiegels

Parkinsonoid, Akathisie, häufig Symptome eines erhöhten Prolaktinspiegels

\section{längerfristige Nebenwirkungen}

Gewichtszunahme, Osteoporose, Absetzsymptome

wie SSRI

Gewichtsverlust, erhöhtes Krampfrisiko

Gewichtzunahme, Absetzsymptome

Gewichtszunahme, Absetzsymptome

wie SSRI

Diätvorschriften beachten!

Spätdyskinesien

Gewichtszunahme, selten Spätdyskinesien

Gewichtszunahme, metabolisches Syndrom

Gewichtzunahme (v. a. Risperidon und Paliperidon), selten Spätdyskinesien

bei langdauernder Hyperprolaktinämie Osteoporose, selten Spätdyskinesien

\section{Stimmungsstabilisierer („Mood Stabilizer“)}

Lithium

feinschlägiger Tremor, Ataxie, Schwindel,

Gewichtszunahme

Kopfschmerzen, Übelkeit, Polydipsie, Polyurie, Diarrhö,

Mundtrockenheit, Ödeme, Verschlechterung

vorbestehender Hauterkrankungen 
- Tab. 1 (Fortsetzung)

\begin{tabular}{|c|c|c|}
\hline Medikamente & Nebenwirkungen zu Therapiebeginn & längerfristige Nebenwirkungen \\
\hline VPA & $\begin{array}{l}\text { vorübergehender Haarausfall, Ödeme, Tremor } \\
\text { (i. d. R. grobschlägiger als unter Lithium), Parästhesien, } \\
\text { Kopfschmerzen, Müdigkeit, Ataxie, Übelkeit, } \\
\text { Hypersalivation, Diarrhö, Magenschmerzen }\end{array}$ & $\begin{array}{l}\text { Gewichtszunahme, Osteoporose, } \\
\text { Menstruationsstörungen }\end{array}$ \\
\hline CBZ & $\begin{array}{l}\text { Sedierung, Schwindel, Ataxie, Störungen der } \\
\text { Okulomotorik (Doppelbilder) }\end{array}$ & $\begin{array}{l}\text { gelegentlich leichte Gewichtszunahme, } \\
\text { Osteoporose }\end{array}$ \\
\hline LTG & $\begin{array}{l}\text { Kopfschmerzen, Schwindel, Schlafstörungen und } \\
\text { Unruhe, Übelkeit, Diarrhö }\end{array}$ & Osteoporose \\
\hline \multicolumn{3}{|l|}{ Hypnotika und Sedativa } \\
\hline BZ & $\begin{array}{l}\text { Kopfschmerzen, Schwindel, paradox verstärkte } \\
\text { Schlaflosigkeit und Unruhe, Diarrhö, Übelkeit, } \\
\text { Bauchschmerzen }\end{array}$ & Toleranzentwicklung, Abhängigkeit \\
\hline $\mathrm{AH}$ & $\begin{array}{l}\text { Schwindel, Benommenheit, Kopfschmerzen, Mund- und } \\
\text { Halstrockenheit, Obstipation, Übelkeit, Diarrhö, } \\
\text { verstopfte Nase, Miktionsstörungen, Akkommodati- } \\
\text { onsstörungen }\end{array}$ & $\begin{array}{l}\text { Toleranzentwicklung, Abhängigkeit, } \\
\text { Rebound-Schlaflosigkeit }\end{array}$ \\
\hline Tryptophan & Schwindel, Kopfschmerzen, Lichtempfindlichkeit & \\
\hline $\begin{array}{l}\text { pflanzliche Sedativa: Baldrian, Passions- } \\
\text { blume, Johanniskraut (bis } 300 \text { mg/d) }\end{array}$ & Magen-Darm-Symptome (z. B. Übelkeit, Bauchkrämpfe) & Fotosensibilisierung (Johanniskraut) \\
\hline \multicolumn{3}{|l|}{ Antidementiva (Nootropika) } \\
\hline Ginkgo biloba & $\begin{array}{l}\text { Kopfschmerzen, Schwindel, leichte } \\
\text { Magen-Darm-Beschwerden }\end{array}$ & \\
\hline $\begin{array}{l}\text { Acetylcholinesterase-Inhibitoren } \\
\text { (Donepezil, Rivastigmin, Galantamin) }\end{array}$ & $\begin{array}{l}\text { Diarrhö, Übelkeit, Appetitlosigkeit, Kopfschmerzen, } \\
\text { Erregungszustände, Schlaflosigkeit }\end{array}$ & \\
\hline NMDA-Antagonisten (Memantin) & $\begin{array}{l}\text { Schläfrigkeit, Verwirrtheit, Schwindel, Kopfschmerzen, } \\
\text { Obstipation }\end{array}$ & \\
\hline Piracetam & $\begin{array}{l}\text { Nervosität, Aggressivität, Schlafstörungen, } \\
\text { Hyperkinesie }\end{array}$ & Gewichtszunahme \\
\hline \multicolumn{3}{|l|}{ Psychostimulanzien } \\
\hline Amphetamin-Derivate & $\begin{array}{l}\text { Nervosität, Infekte der oberen Atemwege, Appetitver- } \\
\text { lust, Affektlabilität, Panikattacken, Tics, Einschlafstö- } \\
\text { rungen, sexuelle Dysfunktion, Bruxismus, Schwindel, } \\
\text { Dyskinesie, Kopfschmerzen, Nausea, abdominelle } \\
\text { Beschwerden, Pruritus, Durst, Änderungen des } \\
\text { Blutdrucks und der Herzfrequenz }\end{array}$ & Gewichtsverlust \\
\hline Atomoxetin & $\begin{array}{l}\text { Hypertonie, Tachykardie, Appetitverlust, Kopfschmer- } \\
\text { zen, Schläfrigkeit, abdominelle und Brustschmerzen, } \\
\text { Übelkeit, Stimmungsschwankungen, Schwindel, } \\
\text { Obstipation, Dermatitis, Schlaflosigkeit, Agitiertheit, } \\
\text { Angst, Tics, Mydriasis, Pruritus, Lethargie, sexuelle } \\
\text { Dysfunktion }\end{array}$ & Gewichtsverlust \\
\hline \multicolumn{3}{|c|}{$\begin{array}{l}\text { Auf die kursiv gesetzten Nebenwirkungen und Möglichkeiten ihrer Behandlung wird im Text näher eingegangen. Achtung: Dass identische Nebenwirkungen } \\
\text { bei verschiedenen Stoffgruppen aufgelistet sind, bedeutet keinesfalls eine vergleichbare Häufigkeit, sondern nur, dass das Auftreten in >1\% der Patienten } \\
\text { beobachtet wurde. SSRI: Serotonin-Wiederaufnahmehemmer, SNRI: Serotonin-Noradrenalin-Wiederaufnahmehemmer, DNRI: Dopamin-Noradrenalin-Wie- } \\
\text { deraufnahmehemmer, TZA: tri- und tetrazyklische Antidepressiva, NaSSA: noradrenerge und spezifisch serotonerge Antidepressiva, MMBA: multimodale } \\
\text { Modulatoren der Rezeptoren und der Wiederaufnahme biogener Amine, MAO-I: Monoaminooxidase-Inhibitoren, MSAD: melatonerg-serotonerg wirksame } \\
\text { Antidepressiva, THA: typische hochpotente Antipsychotika, TMNA: typische mittel- und niederpotente Antipsychotika, AAD: atypische Antipsychotika } \\
\text { (Dibenzepin), AAB: atypische Antipsychotika (Benzisoxazol-Derivate, andere Stoffe), AABA: atypische Antipsychotika (Benzamide), VPA: Valproinsäure-De- } \\
\text { rivate, CBZ: Carbamazepin und Oxcarbazepin, LTG: Lamotrigin, BZ: Benzodiazepine und Derivate (Zolpidem, Zopiclon), AH: Antihistaminika (Diphenhydra- } \\
\text { min, Doxylamin). }\end{array}$} \\
\hline
\end{tabular}


Merke

Generell gelten 2 Empfehlungen bei medikamenteninduzierter Nausea: Einnahme nach den Mahlzeiten und langsamere Aufdosierung.

Bei leichterer Ausprägung von Nausea kann zusätzlich Ingwer helfen; verschiedene verkapselte Präparate mit reinem Ingwer sind frei erhältlich. Empfohlen werden ca. 800 mg 2 - 3-mal täglich. Bei schwererer Ausprägung von Nausea kann je nach Ursache auch kurzzeitig regelmäßig ein postsynaptischer $5-\mathrm{HT}_{3}$-Rezeptorblocker verabreicht werden, z. B. ein „Setron“ wie Ondansetron. Auch ein klassisches Antiemetikum, das zusätzlich die D2-Rezeptoren der Area postrema blockiert, z. B. Metoclopramid, kann indiziert sein.

\section{Cave}

Unter Metoclopramid können Akathisie und auch Dystonien auftreten, und die längerfristige Anwendung kann tardive Dyskinesien hervorrufen.

Ist die Übelkeit durch ein SSRI oder SNRI induziert, kann eine Kombinationsbehandlung mit Mirtazapin helfen: Sie führt zu einer besseren antidepressiven (und schlafinduzierenden) Wirkung und zusätzlich zu einer Abnahme der Übelkeit, da Mirtazapin u. a. 5- $\mathrm{HT}_{3}$-Rezeptoren blockiert.

Selbstverständlich sind organische Ursachen, wie z. B. eine medikamenteninduzierte Pankreatitis oder Hepatitis, auszuschließen, insbesondere bei zusätzlichen Symptomen wie abdominellen Schmerzen.

\section{Appetitverlust}

Über Stimulation serotonerger $5-\mathrm{HT}_{2} \mathrm{C}$-Rezeptoren kann ein Appetitverlust vermittelt werden. Dieser ist dementsprechend bei Behandlung mit SSRI oder Stimulanzien häufiger anzutreffen, kann aber auch bei anderen Antidepressiva auftreten. Allerdings ist Appetitverlust auch ein häufiges Symptom der Depression, weswegen die Eruierung des zeitlichen Zusammenhangs zum Therapiebeginn wichtig ist.

Kurzwirksame Stimulanzien (z. B. Methylphenidat) und Antidepressiva (Bupropion) bewirken den Appetitverlust vermutlich beide über eine Dopamin-Noradrenalin-Wiederaufnahmehemmung. Hier kann es helfen, entweder morgens vor der Einnahme oder spätabends, wenn die Wirkung nachlässt, größere Mahlzeiten einzunehmen und über den Tag zu „snacken“. Bittermittel - z. B. Wermut, Angelika, Löwenzahn, Schafgarbe, Ringelblume oder Enzian - als Tee oder Tinktur wirken appetitanregend. Sie sollten etwa eine halbe Stunde vor den Mahlzeiten eingenommen werden (nicht bei Patienten mit vorbestehender Magenschleimhautreizung). Die zusätzliche Gabe von Mirtazapin, Mianserin oder Agomelatin, die
5- $\mathrm{HT}_{2 \mathrm{C}}$-antagonistisch wirken, kann hilfreich sein. Zudem kann sie Schlafstörungen (aber auch Depressionen und Angststörungen) bei Aufmerksamkeitsdefizit-Hyperaktivitäts-Störung (ADHS) gleich mitbehandeln.

\section{Gewichtszunahme}

Eine nach Therapiebeginn einsetzende Gewichtszunahme kann - insbesondere bei Frauen - die Therapieadhärenz frühzeitig infrage stellen. Wenn nicht adressiert, können langfristig eine deutliche Adipositas und metabolische Probleme die Folge sein. Medikamente mit antagonistischer Aktivität an zentralen $5-\mathrm{HT}_{2} \mathrm{C}^{-}$und $\mathrm{H}_{1}$-Rezeptoren führen über eine Appetitsteigerung oft zu Gewichtszunahme. Hierzu zählen insbesondere trizyklische Antidepressiva (TZA), Mirtazapin und die atypischen Antipsychotika Clozapin, Olanzapin, Quetiapin, Risperidon und Paliperidon. Olanzapin und Clozapin, sowie in geringerem Maße auch Mirtazapin, führen zudem auch zu einem Anstieg von Leptin. Dies kann die Ausbildung einer Leptin-Resistenz zur Folge haben, wie sie oft bei Adipositas beobachtet wird [14].

Eine verstärkte Aktivität des Tumor-Nekrose-Faktor-(TNF-) Systems findet sich bei adipösen Patienten und scheint mit der Adipositas kausal verknüpft [15]. Gewichtssteigernde Psychopharmaka aktivieren das TNF-System schon bevor es zu einer messbaren Veränderung des Körpergewichts kommt.

Neben den genannten Antipsychotika und Antidepressiva sind auch die Stimmungsstabilisierer Lithium und Valproat, und in einem geringeren Ausmaß Carbamazepin, mit einer Gewichtszunahme assoziiert.

Merke

Diätetische Maßnahmen sind in jedem Fall indiziert. Neben einer Verminderung der Kalorienzufuhr kann generell eine Nahrungsumstellung hin zu einer ausgewogeneren Ernährung eingeleitet werden.

Verstärkte sportliche Aktivität ist stets zu empfehlen nicht zuletzt da auch die Erkrankung selbst (Depression oder schizophrene Negativsymptomatik) zu Gewichtszunahme aufgrund verminderter Aktivität führt.

Haben diese Maßnahmen keinen ausreichenden Erfolg, sollte zunächst eine medikamentöse Umstellung auf ein gewichtsneutrales Medikament geprüft werden - im Fall von Antidepressiva z. B. auf einen SSRI, SNRI, Agomelatin oder Bupropion. Für die antipsychotische Therapie sind in diesem Fall die Atypika Amisulprid, Aripiprazol, Lurasidon oder Ziprasidon zu bevorzugen. Als Stimmungsstabilisierer kann zur Phasenprophylaxe bipolarer Depressionen Lamotrigin eine wirksame Alternative darstellen. 
Wenn ein Absetzen/Umsetzen der bestehenden Medikation nicht infrage kommt, kann die adjuvante Gabe eines appetitmindernden oder die Insulin-Sensitivität beeinflussenden Medikamentes überlegt werden (s. „Hintergrund“).

\section{HINTERGRUND}

\section{Medikamentöse Maßnahmen}

Der als Appetitzügler bis 2010 zugelassene SNRI Sibutramin ist aufgrund schwerer Nebenwirkungen in Deutschland nicht mehr im Handel. Synthetische Amphetamin-Abkömmlinge und das pflanzliche Ephedrin weisen ein hohes Abhängigkeitspotenzial und z. T. bedrohliche Auswirkungen auf das HerzKreislauf-System auf. Einige für andere Indikationen zugelassene Medikamente können allerdings hilfreich sein. Geringe Dosen Topiramat (25-50 mg) oder Zonisamid $(400 \mathrm{mg}$ ) haben einen appetitmindernden Effekt, der sich in einer langsamen Gewichtsreduktion niederschlägt. Am besten untersucht ist das orale Antidiabetikum Metformin, das sich bei medikamenteninduzierter Gewichtszunahme in zahlreichen Studien als wirksam erwies. Bei Dosierungen $>750 \mathrm{mg}$ ließ sich zudem neben dem antihyperglykämischen auch ein triglyzeridsenkender Effekt nachweisen [16].

\section{Kopfschmerz}

Kopfschmerz wird häufig als Nebenwirkung - insbesondere von Antidepressiva - berichtet, wobei sich in den meisten klinischen Studien kein signifikanter Häufigkeitsunterschied zwischen Medikament und Placebo abbildet. Dennoch sollte auf den subjektiven Eindruck des Patienten natürlich eingegangen werden und nach organischen Ursachen gesucht werden.

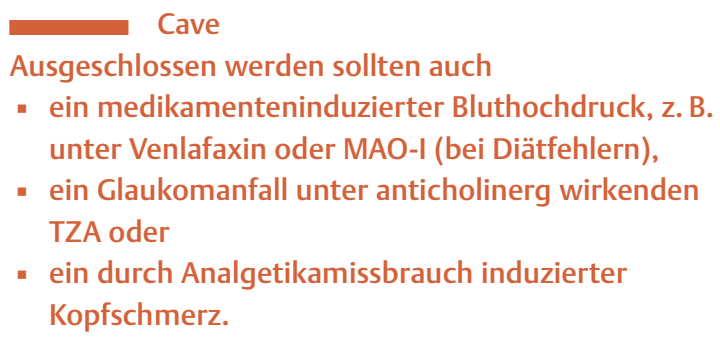

Leichtere Kopfschmerzen können bei Therapiebeginn mit serotonerg stimulierenden Substanzen wie SSRI, SNRI, aber auch Lithium und Lamotrigin vorübergehend auftreten. Dosisreduktion, physikalische Maßnahmen (Stirn kühlen, Frischluft) und Entspannungsverfahren können hier zumeist ausreichend Abhilfe schaffen, sodass auf die Verschreibung eines Schmerzmittels verzichtet werden kann.

\section{Tagesmüdigkeit und Abgeschlagenheit}

Antagonismus an Histamin-( $\left.\mathrm{H}_{1}{ }^{-}\right)$Rezeptoren und 5- $\mathrm{HT}_{2 \mathrm{~A}^{-}}$ Rezeptoren, wie er sich bei einigen atypischen Antipsychotika und Antidepressiva findet, führt oft zu unerwünschter Sedierung und Schläfrigkeit. Dies macht sich aber meist nur zu Therapiebeginn deutlich bemerkbar. Dosisreduktion ist hier nicht immer hilfreich, da oft schon bei geringen Dosen eine maximale Sättigung dieser Rezeptoren besteht (z. B. bei Quetiapin).

Falls die medikamenteninduzierte Müdigkeit persistiert, wäre Therapiewechsel zu einem eher stimulierenden Antidepressivum (z. B. SSRI, SNRI oder Bupropion) bzw. Antipsychotikum (z. B. Aripiprazol, Ziprasidon, Amisulprid) der nächste Schritt.

Schließlich kann an die kurzfristige Zugabe von Modafinil gedacht werden, einer Substanz, die gegen Tagesmüdigkeit bei Schichtarbeit entwickelt wurde. Zusätzlich wurde ein augmentativer Effekt bei der Antidepressivabehandlung festgestellt. Nicht endgültig geklärt ist allerdings ein mögliches Suchtpotenzial, weswegen die Einnahme nur kurzfristig und unter kontrollierten Bedingungen erfolgen sollte.

\section{Mundtrockenheit (Xerostomie)}

Mundtrockenheit ist eine häufige Nebenwirkung von Antidepressiva und Antipsychotika mit anticholinerger Wirkkomponente, tritt aber auch bei bis zu einem Drittel der Patienten unter SSRI auf. Auch unter Lithiumtherapie kann es zu Mundtrockenheit kommen, hier allerdings als Ausdruck einer Dehydration aufgrund von Polyurie. Die kausale Therapie wäre dementsprechend eine Steigerung der Flüssigkeitszufuhr und/oder Adjustierung des Lithiumspiegels.

\footnotetext{
Cave

Neben der subjektiven Unannehmlichkeit können auch Karies und bakterielle Infektionen der Mundhöhle die Folge von Mundtrockenheit sein. Entsprechend ist eine häufigere und verstärkte Mundhygiene wichtig.
}

Hilfreich sind Nahrungsmittel, die den Speichelfluss fördern - z. B. Möhren, Äpfel oder Stangensellerie - sowie zuckerfreie saure Drops, Zitronenbonbons oder Kaugummi. Kleine Schlucke Wasser (ggf. mit etwas Zitronenoder Limettensaft) oder das Lutschen von Eiswürfeln können den Leidensdruck ebenfalls mildern. Für die Nacht kann man von einem Ultraschallvernebler im Schlafzimmer Gebrauch machen.

Sollten diese Schritte nicht zu einer Besserung führen, gibt es eine Reihe von frei verkäuflichen Produkten zur Minderung von Mundtrockenheit und Verbesserung der 
Oralhygiene, z. B. die Biotene ${ }^{\circledR}$-Produktreihe. Ebenso helfen glyzerinbasierte Mundstäbchen und Kaugummi mit Xylitol.

Bei schwerster Mundtrockenheit - und wenn ein Medikamentenwechsel keine Option ist - können cholinerge Medikamente Linderung verschaffen, z. B. Pilocarpin oder Cevimelin, ein Acetylcholinderivat mit erhöhter Affinität für $\mathrm{M}_{1}$ - und $\mathrm{M}_{3}$-Muskarinrezeptoren. Man kann auch ein paar Tropfen Pilocarpin-Augentropfen zu einem Esslöffel Wasser geben, damit den Mund ausspülen und die Mischung anschließend ausspucken. So lassen sich systemische Wirkungen vermeiden.

\section{Übermäßiger Speichelfluss (Hypersalivation)}

Im Vergleich zu Mundtrockenheit ist ein übermäßiger Speichelfluss unter Psychopharmaka eher selten. Bei manchen Medikamenten, z. B. Clozapin oder Olanzapin, ist er aber oft nicht nur lästig, sondern sozial stigmatisierend. Vermutlich wirken diese atypischen Antipsychotika als Agonisten an $\mathrm{M}_{4}$-Rezeptoren, wohingegen sie an den anderen muskarinergen Rezeptoren einen antagonistischen Effekt haben. Weiterhin wirkt Clozapin als Antagonist an adrenergen $\alpha_{1}$ - und $\alpha_{2}$-Rezeptoren. In der Folge steigen die Durchblutung und damit auch die Speichelproduktion. Für Olanzapin wird eine Beteiligung des Tachykinin-Systems als Ursache erhöhter Speichelproduktion diskutiert.

Anticholinerge Substanzen, wie Pirenzepin, Glycopyrrolat, Scopolamin oder Atropin-Tropfen (0,25-0,75 mg sublingual 3-mal täglich), können Linderung verschaffen. Die systemische Anwendung anticholinerger Substanzen erhöht das Risiko eines paralytischen lleus, daher sollte bereits bei beginnender Obstipation mit abführenden Maßnahmen (z. B. Lactulose-Gabe) begonnen werden. Speziell im Fall von Clozapin bietet sich zudem die Kombination mit dem anticholinergen Antipsychotikum Amisulprid an. Dies kann zu einer Reduktion des Speichelflusses und einer potenziell verstärkten antipsychotischen Wirksamkeit führen. Hier sollte eine regelmäßige Kontrolle der QTc-Zeit erfolgen - wie auch bei anderweitiger Polypharmazie mit QTcbeeinflussenden Substanzen. Die lokale Behandlung mit Botulinum-Toxin scheint bisher nur schweren Fällen von Hypersalivation bei neurodegenerativen Erkrankungen (amyotrophe Lateralsklerose, Morbus Parkinson) oder frühkindlicher Zerebralparese vorbehalten.

\section{Exzessives Schwitzen (Hyperhidrosis)}

Schwitzen als Entzugssyndrom bei vorausgegangener Behandlung mit GABAergen Substanzen (z. B. Benzodiazepine) oder Suchtmittelkonsum (Alkohol, Opioide) ist zunächst auszuschließen. Vermehrtes Schwitzen als Nebenwirkung einer Antidepressivatherapie tritt insbesondere unter Bupropion, Venlafaxin, Paroxetin und Citalopram auf [17]. Wechsel des Antidepressivums und
Gebrauch eines antiperspiranten Deosprays kann bereits deutliche Besserung bringen.

Merke

Anders als beim „normalen“ Schwitzen aufgrund von Hitze manifestiert sich Antidepressiva-induziertes Schwitzen hauptsächlich im Kopf- und Brustbereich.

\section{HINTERGRUND}

Innervation von Schweißdrüsen

Schweißdrüsen sind bezüglich ihrer Innervation durch den Sympathikus einzigartig: Die ekkrinen Schweißdrüsen verfügen über cholinerge und die apokrinen über adrenerge Rezeptoren. Entsprechend kann ein medikamentöser Therapieansatz, wenn erforderlich, über eine Blockade postsynaptischer $\alpha_{1}$-Rezeptoren oder postsynaptischer muskarinischer Rezeptoren erfolgen. Eine dritte Variante ist die Blockade präsynaptischer $\alpha_{2}$-Rezeptoren auf hypothalamischer Ebene (Clonidin).

Als erste Option wäre die Kombination mit einem zweiten, $\alpha_{1}$-antagonistischen Antidepressivum wie z. B. Mirtazapin, Mianserin oder Trazodon zu überlegen. Auch für den $\alpha_{1}$-Rezeptorblocker Terazosin (bis maximal $6 \mathrm{mg}$ zur Nacht, langsame Aufdosierung, sonst Gefahr eines Blutdruckabfalls) gibt es Hinweise auf gute Wirksamkeit bei Antidepressiva-induziertem Schwitzen [18]. Alternativ können im Extremfall Clonidin (als präsynaptischer $\alpha_{2}$-Rezeptorantagonist, cave Blutdruckabfall) oder Anticholinergika (Benzatropin oder Glycopyrrolat) versucht werden [19].

\section{Benommenheit und orthostatische Dysregulation}

Die kompensatorische Gegenregulation des Blutdruckes bei einem Abfall aufgrund von Lagewechsel wird über $\alpha_{1}$-Barorezeptoren der großen Blutgefäße vermittelt dementsprechend können alle Medikamente, die den $\alpha_{1}$-Rezeptor blockieren, eine orthostatische Dysregulation hervorrufen. Dazu gehören atypische Antipsychotika (insbesondere Quetiapin, Risperidon und Clozapin), TZA, Trazodon, Mirtazapin, Benzodiazepine, und MAO-Inhibitoren.

\section{Cave \\ Besonders anfällig für orthostatische Dysregulation scheinen ältere Patienten zu sein, vor allem bei gerin- gem Körpergewicht und allgemeiner Gebrechlichkeit.}

Weitere Risikofaktoren sind Dehydrierung, vorausgegangene Bettlägerigkeit, Diabetes mellitus, Morbus Parkinson und gleichzeitige diuretische oder antihypertensive Therapie. Daher sollten zunächst nicht psychiatrische 
Medikamente als Ursache ausgeschlossen werden. Ist von der Kausalität der psychopharmakologischen Behandlung auszugehen und soll diese nicht verändert werden, kann ein Wechsel zur retardierten Form zur Vermeidung von Konzentrationsspitzen bereits helfen. Zusätzlich sollten dem Patienten einige Verhaltenstricks vermittelt werden.

\section{TIPPS FÜR DIE PRAXIS}

Wie lässt sich eine orthostatische Dysregulation vermeiden?

- Abruptes Aufstehen und längeres Stehen ohne Bewegung vermeiden.

- Wenn man stehen muss, Beine kreuzen („Cocktail Posture“) und intermittierend die Beinmuskulatur anspannen, um die venöse Pumpfunktion zu verbessern.

- Bei Auftreten von Benommenheit in die Hocke gehen.

- Generell genug trinken - wenn Durst auftritt, ist dies bereits ein Zeichen beginnender Dehydration.

- Hinreichende Kochsalzzufuhr und Sport, insbesondere Schwimmen ist zu empfehlen [20].

Orthostatische Dysregulation zeigt sich am häufigsten bei Medikamentenauftitrierung und bessert sich unter konstanter Zieldosis.

Stützstrümpfe können hilfreich sein, sie sollten einen zusätzlichen Druck von mindestens $15-20$ mmHg aufbauen. Allerdings nimmt mit der Länge der Strümpfe meist auch die Akzeptanz durch den Patienten ab. Medikamentös kann in schweren Fällen die Gabe von Fludrocortison helfen, vorausgesetzt, der Patient hat keine arterielle Hypertonie oder Herzinsuffizienz [20].

\section{Sexuelle UAW}

Insbesondere unter SSRI sind sexuelle UAW häufig, werden aber oft erst auf gezieltes Befragen berichtet [21] - dabei zählen sie mit zu den Hauptgründen für ein selbstständiges Absetzen der Medikation [22]. Insbesondere bei Frauen werden sie oft ignoriert [23]. Aber nicht immer muss eine Kausalität mit der Psychopharmakotherapie bestehen: Ein Mangel an sexuellem Verlangen sowie erektile Dysfunktion bei Männern findet sich bereits bei $40 \%$ depressiver Patienten unabhängig von der Medikation [24].

Medikamente, die zu einer Erhöhung des Prolaktins über Verminderung von Dopamin (verschiedene Antipsychotika) oder Erhöhung von Serotonin (Antidepressiva) führen, beeinflussen sexuelle Funktionen und Emotionen negativ. Langfristig können auch stimmungsstabilisierende Antiepileptika wie Valproat oder Carbamazepin über
Veränderungen des Testosteron- und Östrogenspiegels die sexuelle Appetenz beeinflussen.

\section{Merke}

Antidepressiva - insbesondere SSRI und SNRI - sowie serotonerg wirkende Medikamente zur Behandlung der ADHS wie Atomoxetin können den sexuellen Erregungsablauf an verschiedenen Stellen behindern, vom sexuellen Verlangen bis zum Orgasmus.

\section{Antidepressiva}

Zunächst stellt sich die Frage, ob die Antidepressivatherapie entbehrlich ist, insbesondere wenn die Patienten bereits wieder remittiert sind. Besteht die Indikation zur Therapiefortführung, findet sich leider nur bei ca. $10 \%$ der Patienten eine spontane Besserung der sexuellen UAW. Ob eine Dosisreduktion eine Option darstellt, hängt von der Schwere der Depression und dem bisherigen Verlauf ab. Ein Wechsel auf einen anderen SSRI bringt meist keine Vorteile, wohingegen andere Wirkstoffklassen oder einzelne Medikamente deutlich weniger mit sexueller Dysfunktion assoziiert sind: z. B. TZA, Bupropion, Mirtazapin, Agomelatin, Duloxetin, Tianeptin, Vortioxetin, Moclobemid.

Kommen Dosisreduktion oder Umsetzen der Medikation nicht infrage, so kann der Versuch einer medikamentösen Linderung der Beschwerden unternommen werden. Gerade bei depressiven Patienten (oder dem zusätzlichen Wunsch nach Nikotinentwöhnung) kann die additive Gabe des Dopamin-Noradrenalin-Wiederaufnahmehemmers Bupropion versucht werden. Allerdings sind die Ergebnisse randomisierter Studien bezüglich einer Besserung SSRI-induzierter UAW uneinheitlich [25]. Bei Frauen wäre ein Versuch mit Bupropion dennoch erste Wahl, da eine Wirksamkeit von Phosphodiesterase-Hemmern bei Patientinnen mit sexuellen UAW nicht nachgewiesen ist [23].

\footnotetext{
Cave

Bupropion hemmt Cytochrom P450 2D6, erhöht also die Blutspiegel von Venlafaxin, Duloxetin, Fluoxetin, Paroxetin und Vortioxetin, was bei einer additiven Gabe zu beachten ist.
}

Bei männlichen Patienten erscheinen die Phosphodiesterase-5-(PDE-5-) Hemmer Sildenafil und Tadalafil bei SSRIinduzierter erektiler Dysfunktion wirksam [25]. Die neu auf den Markt gekommenen Avanafil und Vardenafil wurden diesbezüglich noch nicht untersucht. Tadalafil hat gegenüber Sildenafil den Vorteil der deutlich höheren Spezifität für die PDE-5-Rezeptoren als für PDE-3-Rezeptoren und damit einer höheren Sicherheit bezüglich kardialer UAW. Zudem setzt es aufgrund seiner langen Halbwertszeit von 17 Stunden den depressiven Patienten nicht unter Druck, unmittelbar nach Einnahme sexuell aktiv werden zu müssen - er kann es entspannt angehen. 


\section{Antipsychotika (Prolaktinerhöhung)}

Bei sexueller Dysfunktion unter der Therapie mit einem Prolaktin-erhöhenden Antipsychotikum sollte zunächst eine Dosisreduktion versucht werden. Führt sie zu keiner Besserung, so sollte der Patient umgestellt werden auf ein atypisches Neuroleptikum mit keiner oder nur geringer Wirkung auf Prolaktin. Die beste Evidenz besteht für Aripiprazol, aber auch Quetiapin, Olanzapin, Ziprasidon, Cariprazin, Lurasidon und Clozapin zeigen keine oder nur geringe Auswirkungen auf Prolaktin. Unter additiver Gabe von Aripiprazol wurde sogar eine Besserung sexueller UAW trotz Fortführung des verursachenden Antipsychotikums beschrieben [26]. Treten die sexuellen UAW unter Risperidon depot auf, so kann eine Umstellung auf Paliperidon depot Besserung bringen [27]. Bei männlichen Patienten kann zusätzlich auch die Gabe von PDE5-Hemmern erwogen werden (s. o.).

Ob Flibanserin bei medikamenteninduziertem Libidoverlust bei Frauen wirksam ist, ist bisher nicht untersucht.

\section{Extrapyramidalmotorische Symptome}

Früh- und Spätdyskinesien

Die klassische Akutbehandlung (und Prophylaxe) von Antipsychotika-induziertem Rigor, Hypokinese, Dyskinesien und okulogyrischer Krise stellt die Gabe von Anticholinergika dar, z. B. Biperiden, Benzatropinmesilat oder Procyclidin - in der Akutsituation auch intravenös. Ob sie bei Spätdyskinesien auch wirksam sind, ist umstritten: Es wurden auch Fälle einer deutlichen Verschlechterung von Spätdyskinesien unter langfristiger Anticholinergika-Gabe berichtet [28]. Aus diesem Grund sollten Anticholinergika bei Spätdyskinesien nicht erwogen bzw. abgesetzt werden. Die Empfehlungen der American Academy of Neurology (AAN) lauten, bei Spätdyskinesien zunächst einen Therapieversuch mit Clonazepam oder Gingko biloba zu unternehmen - danach bei geringerer Evidenzlage mit Amantadin oder Tetrabenazin [29]. Eine Umstellung auf besser verträgliche (atypische) Antipsychotika sollte sowohl bei Früh- als auch Spätdyskinesien unbedingt versucht werden, falls die neuroleptische Therapie unverzichtbar erscheint.

\section{Tremor}

Medikamenteninduzierter Tremor tritt eher bei älteren Patienten und meist relativ früh in der Behandlung auf. Eine Unterscheidung von einem genuinen Parkinson-Tremor ist mittels elektrophysiologischer Tremoranalyse möglich [30]. Im Regelfall ist Tremor dosisabhängig, und eine Dosisreduktion sollte zunächst versucht werden. Umstellung auf ein retardiertes Präparat zur Vermeidung von Konzentrationsspitzen und - wenn möglich - Einnahme des Medikaments zur Nacht können ebenfalls versucht werden. Tremorverstärkende Substanzen, z. B. Koffein, sollten vermieden werden.

\section{Cave}

Koffein erhöht die renale Lithiumausscheidung. Reduktion des Kaffeekonsums kann daher bei Lithium-induziertem Tremor zu einer Erhöhung des Lithiumspiegels und damit paradoxerweise zu einer Verstärkung des Tremors führen.

Ist der Tremor so ausgeprägt, dass er den Patienten im Alltag deutlich behindert, kann eine medikamentöse Behandlung versucht werden. Kasuistiken berichten von einer Besserung sowohl von Lithium- als auch Valproatinduziertem Tremor durch Gabe von Propanolol (80 $160 \mathrm{mg} / \mathrm{d}$, in Ausnahmefällen bis $360 \mathrm{mg} / \mathrm{d}$ ). Tritt der Tremor als Teil eines medikamenteninduzierten Parkinsonoids auf, so können auch Anticholinergika oder Amantadin hilfreich sein.

\section{Akathisie}

Akathisie ist eine nur begrenzt willentlich zu unterdrückende, meist quälend empfundene Bewegungsunruhe der Beine. Sie tritt unter antipsychotischer (manchmal auch antidepressiver, insbesondere SSRI) Therapie auf und persistiert, wenn keine Gegenmaßnahmen getroffen werden. Akathisie muss unterschieden werden von einem RestlessLegs-Syndrom, das sich unter Antipsychotikagabe oft erst demaskiert. Dosierungen im höheren therapeutischen Bereich und schnelle Auftitration scheinen das Risiko einer Akathisie zu erhöhen [31]. Entsprechend sollten Dosisreduktion bzw. Präparatewechsel zunächst erwogen werden. Ist dies keine Option, kann die Zugabe von Propanolol (z. B. $40 \mathrm{mg}$ 2-mal täglich) oder eines $5-\mathrm{HT}_{2 \mathrm{~A}}$-Antagonisten (z. B. Mirtazapin) versucht werden. Auch Benzodiazepine sind wirksam bei Akathisie, aber wegen ihres Suchtpotenzials keine wirkliche Alternative für eine länger dauernde Behandlung. Anticholinergika hingegen scheinen bei Akathisie nicht wirksam zu sein [32].

\section{KERNAUSSAGEN}

- Die Psychopharmakotherapie ist bei vielen psychiatrischen Krankheitsbildern unverzichtbar, aber oft mit UAW behaftet.

- Vom Patienten berichtete UAW dürfen nicht ignoriert oder bagatellisiert werden, andernfalls wird dies zu einem selbstständigen Absetzversuch führen.

- Strategien zur Beherrschung der UAW sind abhängig vom Medikament und der Art der Nebenwirkung.

- Stellen Dosisreduktion, Absetzen oder Medikamentenwechsel keine Option dar, so sollten nebenwirkungsspezifische Verhaltensmaßnahmen oder medikamentöse Interventionen zur Anwendung kommen. 


\section{Interessenkonflikte}

Anna Grunze gibt an, dass keine Interessenkonflikte bestehen. Rajnish Mago erhielt in den letzten 3 Jahren Forschungsunterstützung oder Honorare für Beratung oder Vorträge von nachfolgenden Institutionen und Firmen, die z.T. auch in diesem Artikel erwähnte Medikamente vertreiben: Alkermes, Inc.; Allergan, Plc.; Genomind, Inc.; Lundbeck, Otsuka America Pharmaceutical, Inc. und Takeda Pharmaceuticals U.S.A., Inc. Heinz Grunze erhielt in den letzten 3 Jahren Forschungsunterstützung oder Honorare für Beratung oder Vorträge von nachfolgenden Institutionen und Firmen, die z.T. auch in diesem Artikel erwähnte Medikamente vertreiben: Gedeon-Richter, Janssen-Cilag, Lundbeck, Otsuka, Pfizer, Servier.

\section{Über die Autoren}

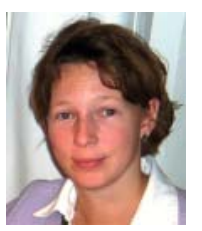

\section{Anna Grunze}

Dr. med. Anna Grunze ist als ärztliche Funktionsbereichsleiterin am Zentrum für Psychiatrie Nordbaden in Wiesloch tätig. Anna.Grunze@pzn-wiesloch.de

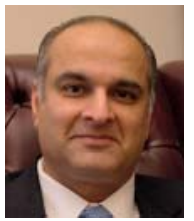

\section{Rajnish Mago}

Dr. med. Rajnish Mago ist Clinical Assistant Professor an der University of Pennsylvania, Philadelphia, Pennsylvania, und Herausgeber von „Simple and Practical Mental Health“. simple@simpleandpractical.com

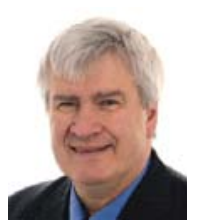

\section{Heinz Grunze}

PD Dr.med. Heinz Grunze ist Chefarzt der Klinik für Allgemeine Psychiatrie und Psychotherapie Ost am Klinikum am Weissenhof in Weinsberg und Dozent an der Paracelsus Medizinischen Privatuniversität Nürnberg. heinz.grunze@gmail.com

\section{Korrespondenzadresse}

Priv. Doz. Dr. Heinz Grunze

Klinik für Allgemeine Psychiatrie und Psychotherapie Ost Klinikum am Weissenhof 74189 Weinsberg heinz.grunze@gmail.com

\section{Literatur}

[1] Fuertes JN, Mislowack A, Bennett J et al. The physician-patient working alliance. Patient Educ Couns 2007; 66: 29-36

[2] Vos T, Flaxman AD, Naghavi M et al. Years lived with disability (YLDs) for 1160 sequelae of 289 diseases and injuries 19902010: a systematic analysis for the Global Burden of Disease Study 2010. Lancet 2012; 380: 2163-2196

[3] Angst F, Stassen HH, Clayton PJ et al. Mortality of patients with mood disorders: follow-up over 34-38 years. J Affect Disord 2002; 68: 167-181

[4] Barth J, Schumacher M, Herrmann-Lingen C. Depression as a risk factor for mortality in patients with coronary heart disease: a meta-analysis. Psychosom Med 2004; 66: 802 - 813
[5] Nicholson A, Kuper H, Hemingway H. Depression as an aetiologic and prognostic factor in coronary heart disease: a metaanalysis of 6362 events among 146538 participants in 54 observational studies. Eur Heart J 2006; 27: $2763-2774$

[6] Fikenzer K, Knoll A, Lenski D et al. Chronische Herzinsuffizienz: Teufelskreis aus geringer Einnahmetreue von Medikamenten und kardialer Dekompensation. Dtsch Med Wochenschr 2014; 139: $2390-2394$

[7] Leucht S, Hierl S, Kissling W et al. Putting the efficacy of psychiatric and general medicine medication into perspective: review of meta-analyses. Br J Psychiatry 2012; 200: 97 - 106

[8] Carvalho AF, Sharma MS, Brunoni AR et al. The safety, tolerability and risks associated with the use of newer generation antidepressant drugs: a critical review of the literature. Psychother Psychosom 2016; 85: 270-288

[9] Wilson E, Lader M. A review of the management of antidepressant discontinuation symptoms. Ther Adv Psychopharmacol 2015; 5: $357-368$

[10] Benkert O, Hippius H. Kompendium der Psychiatrischen Pharmakotherapie. 11. Aufl Heidelberg: Springer; 2017

[11] Gründer G, Benkert O. Handbuch der Psychopharmakotherapie. 2. Aufl Heidelberg: Springer; 2012

[12] Zaudig M, Trautmann-Sponsel RD, Joraschky P et al. Therapielexikon Psychiatrie, Psychosomatik, Psychotherapie. Heidelberg: Springer; 2006

[13] Dunner DL, Wohlreich MM, Mallinckrodt CH et al. Clinical consequences of initial duloxetine dosing strategies: Comparison of 30 and $60 \mathrm{mg}$ QD starting doses. Curr Ther Res Clin Exp 2005; 66: $522-540$

[14] Kraus T, Haack M, Schuld A et al. Body weight and leptin plasma levels during treatment with antipsychotic drugs. Am J Psychiatry 1999; 156: 312-314

[15] Tzanavari T, Giannogonas P, Karalis KP. TNF-alpha and obesity. Curr Dir Autoimmun 2010; 11: 145-156

[16] Zheng W, Li XB, Tang YL et al. Metformin for weight gain and metabolic abnormalities associated with antipsychotic treatment: meta-analysis of randomized placebo-controlled trials. J Clin Psychopharmacol 2015; 35: 499-509

[17] Marcy TR, Britton ML. Antidepressant-induced sweating. Ann Pharmacother 2005; 39: 748-752

[18] Mago R, Thase ME, Rovner BW. Antidepressant-induced excessive sweating: clinical features and treatment with terazosin. Ann Clin Psychiatry 2013; 25: 186-192

[19] Mago R. Glycopyrrolate for antidepressant-associated excessive sweating. J Clin Psychopharmacol 2013; 33: 279-280

[20] Shibao C, Lipsitz LA, Biaggioni I. ASH position paper: evaluation and treatment of orthostatic hypotension. J Clin Hypertens (Greenwich) 2013; 15: 147-153

[21] Cartwright C, Gibson K, Read J et al. Long-term antidepressant use: patient perspectives of benefits and adverse effects. Patient Prefer Adherence 2016; 10: 1401-1407

[22] Clayton AH, Alkis AR, Parikh NB et al. Sexual dysfunction due to psychotropic medications. Psychiatr Clin North Am 2016; 39: $427-463$

[23] Lorenz T, Rullo J, Faubion S. Antidepressant-induced female sexual dysfunction. Mayo Clin Proc 2016; 91: 1280-1286

[24] Kennedy SH, Dickens SE, Eisfeld BS et al. Sexual dysfunction before antidepressant therapy in major depression. J Affect Disord 1999; 56: 201-208

[25] Taylor M], Rudkin L, Bullemor-Day P et al. Strategies for managing sexual dysfunction induced by antidepressant 
medication. Cochrane Database Syst Rev 2013; 5: doi: CD003382

[26] Mir A, Shivakumar K, Williamson RJ et al. Change in sexual dysfunction with aripiprazole: a switching or add-on study. J Psychopharmacol 2008; 22: 244-253

[27] Montalvo I, Ortega L, Lopez X et al. Changes in prolactin levels and sexual function in young psychotic patients after switching from long-acting injectable risperidone to paliperidone palmitate. Int Clin Psychopharmacol 2013; 28: 46-49

[28] Khouzam HR. Identification and management of tardive dyskinesia: A case series and literature review. Postgrad Med 2015; 127: 726 - 737

[29] Bhidayasiri R, Fahn S, Weiner WJ et al. Evidence-based guideline: treatment of tardive syndromes: report of the Guideline Development Subcommittee of the American Academy of Neurology. Neurology 2013; 81: 463 -469
[30] Nistico R, Fratto A, Vescio B et al. Tremor pattern differentiates drug-induced resting tremor from Parkinson disease. Parkinsonism Relat Disord 2016; 25: 100-103

[31] Miller CH, Fleischhacker WW. Managing antipsychotic-induced acute and chronic akathisia. Drug Saf 2000; 22: 73 - 81

[32] Lohr JB, Eidt CA, Abdulrazzaq AA et al. The clinical challenges of akathisia. CNS Spectr 2015; 20: 1-14

Bibliografie

DOI https://doi.org/10.1055/s-0043-110654

Dtsch Med Wochenschr 2017; 142: 1690-1700

(C) Georg Thieme Verlag KG Stuttgart · New York ISSN 0012-0472 